\title{
Screening for Social Determinants of Health in Michigan Health Centers
}

\author{
Elena Byhoff, MD, MSc, Alicia J. Cohen, MD, MSc, Mary C. Hamati, BS, \\ Julie Tatko, MSW, Matthew M. Davis, MD, MAPP, and Renuka Tipirneni, MD, MSc
}

Objective: Through an academic-community partnership with a statewide consortium of health centers (HCs) in Michigan, we characterize the current scope of screening for social determinants of health (SDH).

Methods: We requested copies of forms used to screen for SDH at the $39 \mathrm{HC}$ organizations in Michigan. Using content analysis, we examined variation in screening domains and processes. We present descriptive analyses of $\mathrm{HC}$ characteristics and patient demographics.

Results: We received screening documentation from 23 of the 39 HCs (59\%), representing 167 delivery sites. We found broad empiric consensus regarding a core set of $13 \mathrm{SDH}$ screening domains that align with nationally recommended screening guidelines. Two additional domains, Culture and Functional Status, were screened for by $<40 \%$ of HCs. While patient self-report is the most frequent mode of SDH screening (41\%), many HCs use staff members to administer the screening documents.

Conclusions: HCs across a large and diverse state are screening for SDH and largely agree on core SDH screening domains. Using existing empiric data from frontline providers can inform potential best practices in SDH screening. (J Am Board Fam Med 2017;30:418-427.)

Keywords: Community Health Centers, Michigan, Screening, Social Determinants of Health

Poor health associated with unmet social need is a local, state, and national problem. Individuals living in socioeconomically disadvantaged circumstances are at increased risk of developing chronic medical conditions, face greater barriers to successful disease management, and experience higher rates of

This article was externally peer reviewed.

EB and AJC are co-first authors.

Submitted 27 February 2017; revised 27 February 2017; accepted 5 March 2017.

From the Department of Medicine, Tufts University School of Medicine, and the Institute for Clinical Research and Health Policy Studies, Tufts Medical Center, Boston, MA (EB); the Department of Family Medicine (AJC), the Institute for Healthcare Policy \& Innovation (AJC, RT), and the Division of General Medicine (RT), University of Michigan, Ann Arbor; the Center for Clinical Management Research, Department of Veterans Affairs, Ann Arbor, MI (AJC); the Michigan State University College of Human Medicine, East Lansing (MCH); the Michigan Primary Care Association, Lansing (JT); the Departments of Pediatrics and Medical Social Sciences, Northwestern University Feinberg School of Medicine, Chicago, IL (MMD); the Ann \& Robert H. Lurie Children's Hospital of Chicago, Chicago, IL (MMD).

Funding: This research was funded in part by the Michigan Institute for Clinical \& Health Research (grant UL1TR000433), and by the Robert Wood Johnson Foundation Clinical Scholars Program (EB and AJC). both emergency department utilization and inpatient hospital admissions. ${ }^{1-6}$ Recognizing the importance of social determinants of health (SDH) to health outcomes, utilization, and costs, the $\mathrm{Na}$ tional Academy of Medicine, Centers for Disease Control and Prevention, and Centers for Medicare \& Medicaid Services have all called for evidencebased initiatives to better screen for and address SDHs in clinical settings. ${ }^{7-9}$

While growing evidence informs instruments used to screen for $\mathrm{SDH},{ }^{10-16}$ little is known about current screening practices for $\mathrm{SDH}$ in clinical settings. Health centers (HCs), including federally qualified HCs (FQHC), have focused on the importance of addressing SDH since their origins in the 1960s. Over $9000 \mathrm{HC}$ delivery sites currently serve $>24$ million Americans who have dispropor-

Conflict of interest: none declared.

Corresponding author: Elena Byhoff, MD, MSc, Institute for Clinical Research and Health Policy Studies, Tufts Medical Center, 800 Washington St, Box 63, Boston, MA 02111 (E-mail: ebyhoff@tuftsmedicalcenter.org). 
tionately high rates of unmet social needs, health disparities, and poor health outcomes. ${ }^{17,18}$

To our knowledge, no peer-reviewed publications examine the scope of SDH screening practices in primary care settings in general or in HCs specifically_where health care providers may be at the forefront of integrating SDH considerations into practice. To address this knowledge gap, an academic-community partnership was forged between researchers at the University of Michigan and the Michigan Primary Care Association, a statewide consortium of HCs in Michigan. Together, we examined (1) current screening practices for SDH across Michigan HCs, including the number and types of SDH domains screened; and (2) settings in which screening was administered, and by whom.

\section{Methods}

\section{Data Sources}

The study team requested copies of all forms used to screen for SDH at the $39 \mathrm{HC}$ organizations in Michigan, representing 240 delivery sites across the state (Supplementary Figure 1). All HCs were either FQHCs (grantees of the Health Center Program that receive grants under section 330 of the Public Health Service Act) or FQHC "look-alikes" (Health Center entities that are determined by the U.S. Department of Health and Human Services to be eligible to receive funding under the Health Center Program but do not). ${ }^{19} \mathrm{We}$ also collected information from each $\mathrm{HC}$ organization regarding (1) when during the clinic visit each screening form is administered, (2) who completes the documentation, (3) types of visits during which screening takes place, (4) where screening information is documented, and (5) specific patient populations targeted for screening (if applicable). We obtained data about $\mathrm{HC}$ characteristics, resources, and patient demographics from the Uniform Data System; this system aggregates annual data reported by all HCs funded by the federal Health Resources and Services Administration on a variety of operational and performance measures, including patient demographics, services provided, staffing, clinical indicators, utilization rates, costs, and revenues. ${ }^{19}$ All HCs (both grantees and look-alikes) are required to report annually to the Uniform Data System. ${ }^{19,20}$

This research did not involve patient data. This study was deemed exempt from human subjects review by the University of Michigan Institutional Review Board.

\section{SDH Domain Development}

Using recommended SDH domains from the National Academy of Medicine, Centers for Disease Control and Prevention, and World Health Organization as a starting point, we used an iterative deductive approach to generate an empiric set of core SDH screening domains and subdomains, which we then used to code all collected screening forms. ${ }^{7,9,21,22}$ We first reviewed national initiatives, consensus statements, and expert recommendations regarding SDH screening in order to identify existing screening domains. We then piloted and revised our preliminary SDH domain codes using a sample subset of screening forms in order to create a comprehensive coding scheme for identifying and categorizing SDH screening questions. "Core domains" represented large cohesive themes that comprised more specific or detailed screening questions. Frequently used and more specific screening items were considered subdomains.

\section{Coding and Content Analysis}

Content analysis is a systematic means of describing and quantifying written, visual, or verbal data into parsimonious categories that can be analyzed with statistical methods. ${ }^{23,24}$ It is also frequently used as a method for analyzing documents whose primary intent is not to collect data for analytic purposes. ${ }^{23}$ Three coders (EB, AJC, MCH) used content analysis to assign $\mathrm{SDH}$ domains to each screening document. A minimum of 2 coders independently reviewed $65 \%$ of all screening documents. Concordance for code assignments between each coding team pair was $\geq 95 \%$. All 3 coders collectively reviewed any differences in coding, which were resolved through consensus.

We decided a priori that any document or question with the apparent sole purpose of medical care or billing was not considered an SDH screening item per se, but rather part of the routine services provided by the HC. Examples of excluded documents include forms screening for sexually transmitted infections and questions about immunization history or autism spectrum disorder. While these documents may include questions that could be coded using our SDH domains, the intent of these types of screening is diagnostic, rather than to identify social needs. Documents that were used to 
determine financial resources explicitly for billing purposes, such as a sliding-fee application, were also excluded from our SDH coding analysis. We included items as having an SDH screening intent if they were asked in the context of other social circumstances, rather than in a medical context. For example, in a section that included questions about living situation, nursing home residency, and roommates, a question in the same section asking about functional status or recent falls would be coded as an SDH screening item.

Codes were assigned to individual screening questions, subsections of documents screening for particular needs, or entire documents with a single screening intent. As an example, many HCs submitted forms that included evidenced-based screening instruments for depression and anxiety. ${ }^{25-27}$ While these documents include individual questions within a given instrument or scale that could each have received separate codes, because these scales are intended to be administered as a unit, we instead assigned a single code to the entire scale. For example, the 9-item Patient Health Questionnaire is a validated depression screen that includes a range of questions about mood, sleep, energy, agitation, appetite, concentration, and suicidality; we assigned to all 9 questions in the document a single code for depression screening.

\section{Results}

\section{HC Characteristics}

We analyzed the content of screening documents from 59\% of HCs ( $n=23$ ); these represented 167 delivery sites (mean number of delivery sites per HC organization, 8; range, 1-25) across the state of Michigan who responded to our request for screening documents. Characteristics of responding HCs and their corresponding patient populations are presented in Table 1 . We received screening forms from HCs representing all geographic regions of the state. Of the participating HCs, 6 (26\%) had delivery sites in rural locations (based on ZIP code), all of which were in the northern and middle regions of the state. A total of $16 \mathrm{HCs}(70 \%)$ were in urban settings, and $1 \mathrm{HC}(4 \%)$ provided care in both rural and urban areas. The HCs employed a mean of 9.0 full-time equivalent physicians (range, $0-21.6$ ) and a mean of 5.3 full-time equivalent mental health providers (range, 0-48.1), who included substance use disorder counselors, psychia- trists, licensed clinical psychologists, licensed clinical social workers, and other mental health staff.

Responding HCs had a total of $1,118,557$ medical visits (mean, 50,844; range, 1,342-96,236) and 96,377 mental health visits (mean, 4,381; range, $0-36,495)$ in 2014 , corresponding to 459,313 unique patients- or $75 \%$ of all patients seen by HCs in Michigan in 2014. The patient population served by responding HCs was $56 \%$ female, and the majority was non-Hispanic white (56\%). Over two thirds $(70 \%)$ of patients had incomes $\leq 200 \%$ of the federal poverty level; $51 \%$ had health insurance coverage from Medicaid, the Children's Health Insurance Program, or other governmentsponsored coverage, and $21 \%$ were uninsured. A total of $4 \%$ of the patient population represented was homeless. Notably, important differences were found in mental health services, insurance status, racial and ethnic composition, and rural/urban representation between geographic regions in our HC sample. Medical services provided and prevalence of homelessness showed similarities across regions.

\section{Current SDH Screening Practices}

Table 2 presents the 15 core domains identified empirically from all submitted HC screening documents. Those domains are (in alphabetical order) Culture, Demographics, Economic Indicators, Education, Employment Status, Family/Living Arrangements, Functional Status, Health Care Access, Health-Related Behaviors, Language, Material Hardship, Mental Health, Social Support, Trauma/Violence, and Veteran Status. Broadly, these core domains demonstrate substantial alignment with multiple national initiatives to identify best practices for screening for SDH. ${ }^{7,9,21,22,28}$ However, we identified 1 core domain (Culture) that was not represented in any of the referenced national initiatives (Table 2).

Participating HCs screened for a mean of 11 of the 15 core domains (range, 6-15) (Figure 1). In addition to the core domains, we identified 102 subdomains (Table 2). All responding HCs screened for 4 core domains: Employment Status, Mental Health, Family/Living Arrangements, and Demographics. The majority of participating HCs also screened for Language $(\mathrm{n}=22 ; 96 \%)$, Material Hardship $(\mathrm{n}=22$; 96\%), Veteran Status (n $=21 ; 96 \%)$, Economic Indicators ( $\mathrm{n}=21 ; 91 \%)$, Education $(\mathrm{n}=21$; 91\%), Health Care Access $(\mathrm{n}=22 ; 91 \%)$, Social 
Table 1. Characteristics of Health Center Organizations and Patients in the Study Sample

\begin{tabular}{|c|c|c|c|c|c|c|}
\hline \multirow[b]{2}{*}{ Uniform Data System 2014 Data } & \multicolumn{4}{|c|}{ HC Organizations, by Geographic Region } & \multicolumn{2}{|c|}{ All HC Organizations } \\
\hline & $\begin{array}{l}\text { Northern } \\
(\mathrm{n}=5)\end{array}$ & $\begin{array}{l}\text { Middle } \\
(\mathrm{n}=6)\end{array}$ & $\begin{array}{l}\text { Southern } \\
(\mathrm{n}=5)\end{array}$ & $\begin{array}{l}\text { Detroit Metro } \\
\quad(\mathrm{n}=7)\end{array}$ & Total & Mean \\
\hline \multicolumn{7}{|l|}{ HC Characteristics } \\
\hline Delivery sites, $\mathrm{n}$ & 37 & 61 & 41 & 28 & 167 & 7.26 \\
\hline Rural, n (\%) (based on zip code) & $33(89)$ & $18(30)$ & $0(0)$ & $0(0)$ & $51(31)$ & \\
\hline \multicolumn{7}{|l|}{ Total FTEs, $\mathrm{n}$} \\
\hline Primary care ${ }^{*}$ & 38.0 & 42.2 & 31.0 & 34.8 & 146.0 & 6.64 \\
\hline Physicians $^{\dagger}$ & 44.8 & 56.2 & 50.5 & 47.0 & 198.6 & 9.0 \\
\hline Mental health ${ }^{\ddagger}$ & 17.1 & 64.2 & 21.4 & 13.6 & 116.3 & 5.3 \\
\hline \multicolumn{7}{|l|}{ Visits } \\
\hline Medical services & 258,472 & 377,146 & 311,797 & 171,142 & $1,118,557$ & 50,844 \\
\hline Mental health services & 14,694 & 55,073 & 17,645 & 8,965 & 96,377 & 4,381 \\
\hline \multicolumn{7}{|l|}{ Patient population characteristics } \\
\hline Patients served, $\mathrm{n}$ & 82,705 & 159,406 & 130,440 & 86,762 & 459,313 & 20,878 \\
\hline \multicolumn{7}{|l|}{ Race, n (\%) } \\
\hline Asian & $399(1)$ & $1,797(1)$ & $2,502(2)$ & $712(1)$ & $5,410(1)$ & 246 \\
\hline $\begin{array}{l}\text { Native Hawaiian/other Pacific } \\
\text { Islander }\end{array}$ & $49(0)$ & $508(0)$ & $807(1)$ & $392(1)$ & $1,756(0)$ & 80 \\
\hline Black/African American & $445(1)$ & $37,636(24)$ & $34,826(27)$ & $51,022(59)$ & $123,929(27)$ & 5,633 \\
\hline American Indian/Alaskan Native & $325(0)$ & $678(0)$ & $424(0)$ & $193(0)$ & $1,620(0)$ & 74 \\
\hline White & $78,492(95)$ & $96,843(61)$ & $66,558(51)$ & $15,926(18)$ & $257,819(56)$ & 11,719 \\
\hline$>1$ race & $453(1)$ & $8,272(9)$ & $1,619(1)$ & $535(1)$ & $10,879(2)$ & 495 \\
\hline Other & $2,542(3)$ & $13,672(9)$ & $23,704(18)$ & $17,982(21)$ & $57,900(13)$ & 2,632 \\
\hline Hispanic/Latino ethnicity & $691(1)$ & $35,843(23)$ & $22,476(17)$ & $15,903(18)$ & $74,913(16)$ & 3,405 \\
\hline Female sex, \% (mean $\pm \mathrm{SD})$ & $53.9 \pm 1$ & $55.4 \pm 4$ & $57.8 \pm 2$ & $56.0 \pm 8$ & $55.8 \pm 1$ & \\
\hline $\begin{array}{l}\text { Primary language is English, \% } \\
\text { (mean } \pm \text { SD) }\end{array}$ & $99.8 \pm 0$ & $88.4 \pm 13$ & $92.0 \pm 8$ & $86.4 \pm 19$ & $39,193 \pm 92$ & \\
\hline \multicolumn{7}{|l|}{ Special populations, n (\%) } \\
\hline Agricultural workers or dependents & $199(0)$ & $3,808(2)$ & $8,678(7)$ & $492(1)$ & $13,177(3)$ & 599 \\
\hline Homeless & $380(1)$ & $9,185(6)$ & $7,033(5)$ & $3,594(4)$ & $20,192(4)$ & 918 \\
\hline Veterans & $1,272(2)$ & $2,613(2)$ & $1,163(1)$ & $281(0)$ & $5,329(1)$ & 242 \\
\hline Public housing patients & $2(0)$ & $66(0)$ & $534(0)$ & $2,522(3)$ & $3,124(1)$ & 142 \\
\hline School-based patients & $2,593(3)$ & $12,033(8)$ & $8,506(7)$ & $371(0)$ & $23,503(5)$ & 1,068 \\
\hline Total $^{\S}$ & $4,446(5)$ & $27,705(17)$ & $25,914(20)$ & $7,260(8)$ & $65,325(14)$ & 2,969 \\
\hline $\begin{array}{l}\text { Income } \leq 200 \% \text { of the federal poverty } \\
\text { level, } \mathrm{n}(\%)\end{array}$ & 49,078 (59) & $109,944(69)$ & $99,285(76)$ & $64,299(74)$ & $322,606(70)$ & 14,664 \\
\hline \multicolumn{7}{|l|}{ Insurance } \\
\hline $\begin{array}{l}\text { Medicaid/CHIP/other public } \\
\text { insurance }\end{array}$ & $22,065(27)$ & $82,772(52)$ & $76,170(58)$ & $54,115(62)$ & $235,122(51)$ & 10,687 \\
\hline Uninsured & $6,840(8)$ & $40,432(25)$ & $26,858(21)$ & $21,733(25)$ & $95,863(21)$ & 4,357 \\
\hline Medicare & $19,449(24)$ & $15,369(10)$ & $11,785(9)$ & $4,071(5)$ & $50,674(11)$ & 2,302 \\
\hline Private & $34,351(42)$ & $20,827(13)$ & $15,615(12)$ & $6,520(8)$ & $77,313(17)$ & 3,514 \\
\hline
\end{tabular}

Data are number, number (\%), or mean \pm standard deviation, unless otherwise indicated. Based on Uniform Data System (UDS) and Michigan Health Center organizations grouped by geographic region (see Supplementary Figure 1).

*Includes family physicians, internists, and general practitioners.

${ }^{\dagger}$ Includes total primary care and pediatric physicians, obstetricians/gynecologists, and other specialty physicians.

${ }^{\ddagger}$ Includes substance use disorder counselors, psychiatrists, licensed clinical psychologists, licensed clinical social workers, other licensed mental health providers, and other mental health staff.

${ }^{\S}$ Total special populations include agricultural workers or dependents, homeless population, school-based patients (HCs located within a school, often a partnership between a community HC and school), veterans, and public housing patients.

CHIP, Children's Health Insurance Program; FTE, full-time equivalent. 
Table 2. Comparison of Empirically Identified Social Determinant of Health (SDH) Domains from Health Center Screening Documents Compared with Other Recommended SDH Data Collection Domains

Empirically Identified Domains from Health $\quad$ National Quality ${ }^{*}$ Meaningful

Centers* $^{*}$ NAM $^{\dagger}{\text { HP2 } 2020^{\ddagger}}^{\text {Nation }}$ Forum $^{\S}$ USPSTF" WHO ${ }^{\mathbb{I}}$ UDS $^{\#}{\text { Use Stages } 2 / 3^{* *}}^{*}$

Culture (religious/spiritual affiliations or practices)

Demographics (gender/sex, place of birth, sexual orientation, race/ethnicity)

Economic indicators (income, assets, assistance programs, indebtedness)

Education (educational attainment, basic literacy, health literacy, numeracy)

Employment status (unemployment, OSHA, migrant/seasonal or farm worker, day laborers, disability status, mobility status, retirement status, student, unpaid caretaker, active military)

Family/living arrangements (marital status, dependents, children, elders, who lives in the home)

Functional status (ADLs/IADLs, frailty)

Health-related behaviors (alcohol use, caffeine use, drug use, tobacco use, secondhand smoke, physical activity, sexual activity, diet, safety, bike helmets, baby-proof, seat belts, smoke detectors, guns in the home, driving safety, screen time)

Health care access (insurance status, affordability, usual source of care)

Language (primary language, English proficiency, interpreter, other language proficiency)

Material hardship (food insecurity, housin insecurity, utilities, transportation, medication affordability, access to technology, child care, legal services)

Mental health (depression, anxiety, PTSD, ADD/ADHD, suicide risk, stress, caregiver stress, burnout, sleep behaviors)

Social support (community activities, safe environment, environmental exposures, personal safety, bullying, public spaces, racism, discrimination, distrust, school culture)

Trauma/violence (intimate partner violence, trauma, physical abuse, sexual abuse, mental abuse, child abuse)

Veteran status (history of military trauma, combat veteran)

*Bold items represent the core domains; subdomains are set in parentheses.

${ }^{\dagger}$ National Academy of Medicine (NAM) Capturing Social and Behavioral Domains and Measures in Electronic Health Records: Phase 2 . $^{7}$

${ }^{\ddagger}$ Healthy People 2020. ${ }^{9}$

${ }^{\S}$ National Quality Forum, Risk Adjustment for Socioeconomic Status or Other Sociodemographic Factors. ${ }^{21}$

"U.S. Preventive Services Task Force (USPSTF) published recommendations. ${ }^{29}$

${ }^{\mathbb{I}}$ World Health Organization (WHO), A Conceptual Framework for Action on the Social Determinants of Health. ${ }^{22}$

"Uniform Data System (UDS) reporting measures. ${ }^{19}$

${ }^{* *}$ Secretary of Health and Human Services, Health Information Technology Certification Criteria, 2015 edition. ${ }^{30}$

ADD, attention deficit disorder; ADHD, attention deficit and hyperactivity disorder; ADL, activity of daily living; HP2020, Healthy People 2020; IADLs, instrumental activity of daily living; OSHA, Occupation Safety and Health Administration; PTSD, posttraumatic stress disorder. 
Figure 1. Percentages of health centers screening for core domains.

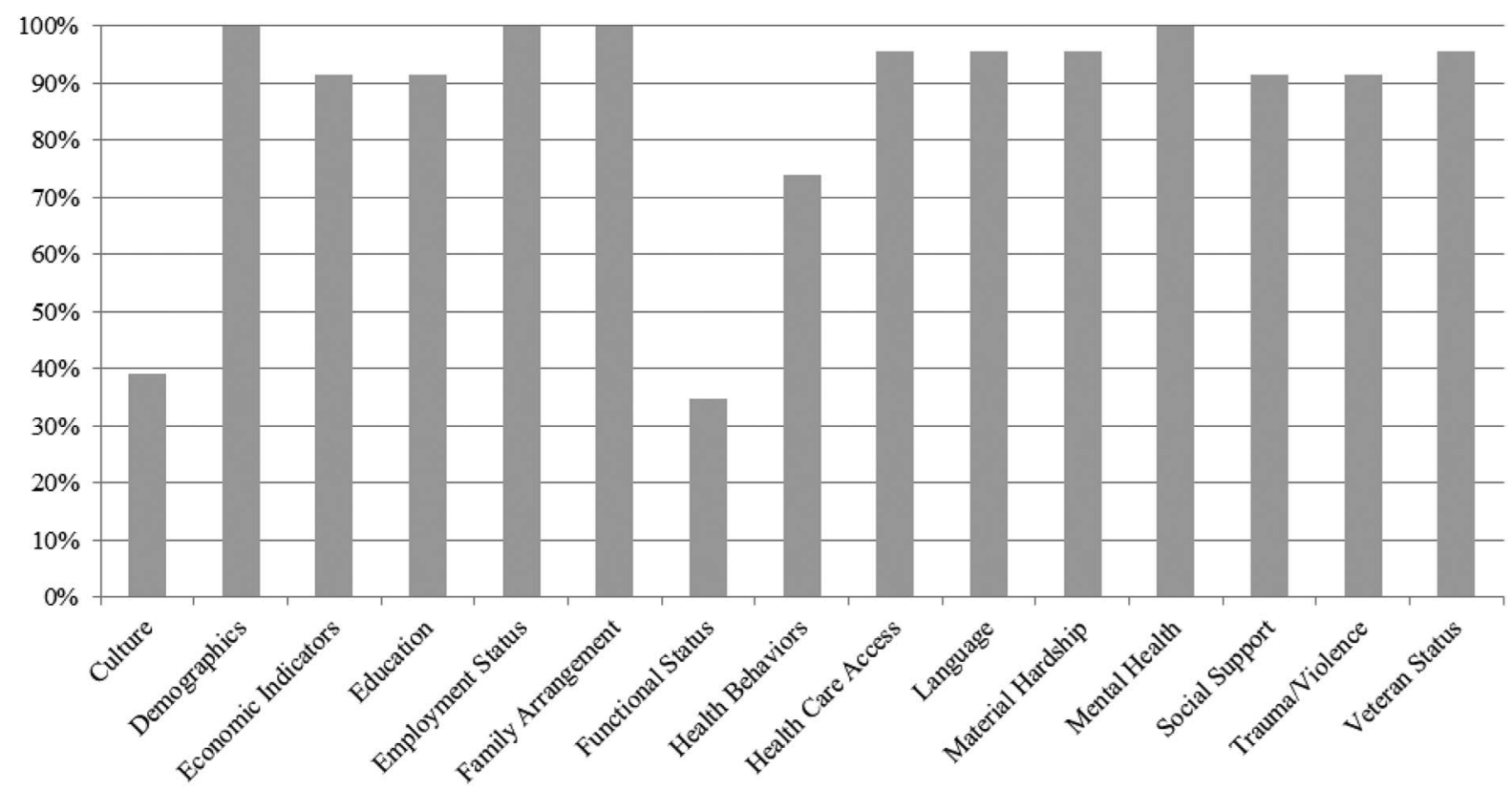

Support $(\mathrm{n}=21 ; 91 \%)$, Trauma/Violence $(\mathrm{n}=21$; $91 \%$ ), and Health-Related Behaviors ( $\mathrm{n}=17$; $74 \%)$. The domains least frequently included in our screening sample were Culture $(\mathrm{n}=9 ; 39 \%)$ and Functional Status $(\mathrm{n}=8 ; 35 \%)$. HCs screened for a mean of 48 subdomains (range, 21-98); the number of subdomains included in the HCs' screening forms varied. Supplementary Table 1 online outlines the number of and variation in screening for all subdomains identified across participating HCs. Certain subdomains were universally included by all HCs that screened for the corresponding core domain.

\section{Variation in Screening Processes}

Modes of SDH screening varied across our sample (Figure 2). HCs (41\%) most frequently reported that information was self-reported by either the patient or the patient's parent. For the majority of $\mathrm{HCs}$, there was a wide representation of staff and provider team members (eg, "other" personnel [24\%], medical assistants [22\%], social workers/ case managers [18\%], health care providers [16\%], front desk staff [12\%], registered nurses [10\%]) who also administered screening forms to patients and parents. Information was collected with equivalent frequency (40\%) before, during, or after a visit. New patients were most frequently targeted for screening $(52 \%)$, but screening was also per- formed $45 \%$ of the time for returning patients and $34 \%$ of the time "as needed." Most of the HCs (63\%) used an electronic health record to record screening results directly, and another 37\% scanned completed paper forms into the electronic health record. Adult patients were more often targeted for SDH screening across all participating HCs $(71 \%)$ than were children, including both pediatric and adolescent visits (19\%). The remaining populations screened by HCs were considered "other," which includes prenatal visits (11\%). Many documents were used in $>1$ category of screening and were not mutually exclusive, so totals may sum to $>100 \%$.

\section{Discussion}

This study is, to our knowledge, the first of its kind to investigate systematically how SDH screening is incorporated into routine clinical practice at sites likely to provide care to predominantly disadvantaged patient populations. Our assessment of current SDH screening practices across Michigan demonstrates that HCs across the state are routinely implementing SDH screening into clinical care across many domains, and that there is broad de facto consensus regarding core domains, despite regional differences in the populations served and the services available. Further, the 15 core domains we empirically identified are largely aligned with 
Figure 2. Health center data collection survey results. Categories are not mutually exclusive and may total $>100 \%$. EHR, electronic health record; MA, medical assistant; RN, registered nurse.

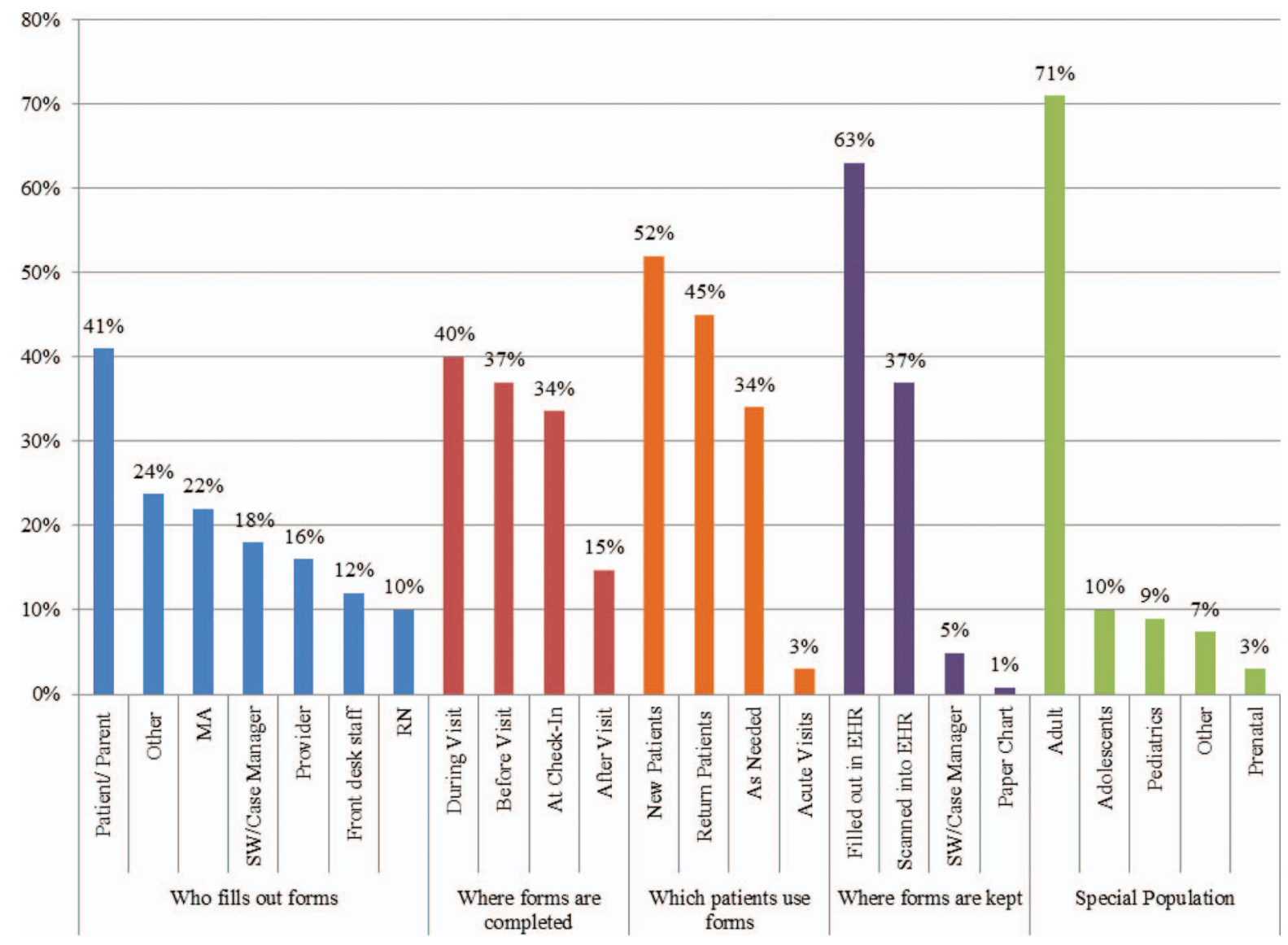

recommended screening guidelines established by multiple national and expert bodies. ${ }^{7,9,21,22,28-30}$ We found variation across HCs, however, with regard to (1) the mode of screening administration and (2) the range of subdomains included. Future work should focus on understanding how and why screening processes differ between HCs, and what, if any, relationship the variation in screening administration has to local populations or resource availability.

Among the 15 core domains identified, not all were routinely included in screening. In fact, only 4 (Demographics, Employment Status, Family and Living Arrangements, Mental Health) were included in the screening documents submitted by all HCs in our sample. Among all responding HCs, $74 \%$ screened for health-related behaviors, which included subdomains to screen for alcohol use, tobacco use, or drug use (Table 2). The lack of universal screening for health-related behaviors was surprising, given the existence of evidencebased instruments to screen specifically for alcohol and tobacco use, ${ }^{31,32}$ as well as expert panel recommendations to screen for these issues in a clinical setting. ${ }^{29}$ It is possible that HCs that did not include items regarding health-related behaviors in the screening forms they submitted, with the implicit expectation that these topics are addressed as part of the clinical visit. Further work to understand how HCs, providers, and other members of the care team define and screen for SDH will be important in generating consensus for $\mathrm{SDH}$ screening instruments. Moreover, clarity in common approaches to screening for SDH can facilitate the efforts of public health officials and policymakers who wish to advance SDH principles and practices.

While increasing evidence suggests that screening for SDH uncovers previously unidentified needs and increases referrals to social services, ${ }^{33,34}$ evidence is still lacking to link screening for SDH with improvements in health outcomes. Concerns exist that screening for social issues that cannot be addressed may cause unintended harm in the doc- 
tor-patient relationship. ${ }^{35}$ Thus, evidence is still needed to assess the association of screening for SDH with patient health outcomes and quality of life. Yet, as SDH increasingly move under the umbrella of health and health care, it will be important to ensure screening practices are methodologically sound and evidence-based.

The identification of core domains already in use in HCs across a large and diverse state is an important first step toward a goal of a unified approach to SDH screening for large-scale implementation. Before this study, 3 state-initiated concurrent projects were being implemented to better understand and address issues related to $\mathrm{SDH}$ in Michigan. ${ }^{36-38}$ Yet limited knowledge was available regarding how relevant SDH data were being collected. Both in Michigan and nationally, consistent measures are greatly needed to monitor SDH across a national population of differing communities, values, and resources. ${ }^{15}$ Selecting these measures will be critically important to ensure proper monitoring of and feedback on how HCs and other providers identify needs and take necessary action steps to improve health. Core domains will allow for trackable metrics across communities to monitor individual SDH, population health, community needs, available ancillary services, and allocation of resources. Identifying core domains will allow policymakers and community stakeholders to determine whether new programs are having their intended health impacts. Without consistent measures across communities, the ability to monitor the influence of SDH on health outcomes, health care utilization, and costs will be limited. In addition, uniform core measures allow for increasing leverage of SDH data in the current landscape of changing payment reforms.

\section{Limitations}

Our study had several limitations. First, our data were collected at the HC organization level. Each $\mathrm{HC}$ organization is responsible for managing and overseeing multiple delivery sites. While the HC organization has specific knowledge of overall policy and procedures across sites, the use and incorporation of screening documents in the workflow may vary at each individual delivery site, and we were unable to capture this variation.

Second, likely some variation existed in $\mathrm{HC}$ organizations' interpretation of what constituted an SDH screening document. The number of screen- ing documents submitted varied across HCs, as did the nature of the submitted documents. Some HCs submitted a large number of medically related screening forms in addition to $\mathrm{SDH}$ screening forms, whereas others submitted only documents related to an ongoing demonstration project in the state that uses an evidence-based questionnaire. ${ }^{36}$ Therefore, our data are limited by how each HC interpreted the request for screening forms.

In addition, our data were collected from FQHC and FQHC look-alikes, rather than all primary care practices in the state. While this may limit the generalizability of our findings across all types of medical practices, HCs serve the highest proportion of low-income patients at risk for unmet social needs. ${ }^{39}$ Therefore, examining SDH screening practices at FQHCs and similar delivery sites is a relevant starting point for gathering data about evidence-based screening practices.

Our data were gathered in one Midwestern state. While Michigan is a large state with HCs that serve a broad mix of urban, rural, tribal, and migrant communities, it is possible that the practices of HCs in one state may not be generalizable to other states. Finally, we present a descriptive analysis of a sample of HCs and their patient populations and resources by geographic region, rather than examine or suggest associations between screening practices and HC demographics. To investigate such associations would require a larger sample of HCs.

\section{Conclusions}

The National Academy of Medicine guidelines represent foundational work assembling a core set of brief, standardized measures for SDH screening. ${ }^{7}$ Our work suggests that many of these recommendations are already in practice in many HCs across Michigan. Given the increasing emphasis on addressing community health needs by Medicare, Medicaid, and other commercial payers, ${ }^{8,40}$ there is an urgent need to develop best practices for identifying and addressing SDHs. Understanding the current landscape of screening for SDHs in a large and diverse state is an important first step toward identifying which SDH data may be most relevant to collect and understanding how best to collect them. Recognizing that HCs are providing health care to a large proportion of at-risk individuals and communities, the core domains identified are 
aligned with recommendations by national expert panels, and they provide a useful starting point for building consensus about the necessary components of SDH screening and how best to incorporate evidence-based items into successful current practice. Future work should focus on understanding variation in screening across practices, how screening may be influenced by both perceived patient needs and the availability of $\mathrm{HC}$ and community resources, approaches to addressing unmet needs once they are identified, and how screening may be used to improve clinical care delivery and population health outcomes.

The authors thank the Michigan Primary Care Association, in particular Kim Sibilsky, Lydia Starrs, and Joe Tran, for providing invaluable help. Thank you to all participating Health Centers in the state of Michigan.

To see this article online, please go to: bttp://jabfm.org/content/ 30/4/418.full.

\section{References}

1. Barnard LS, Wexler DJ, DeWalt D, Berkowitz SA. Material need support interventions for diabetes prevention and control: a systematic review. Curr Diab Rep 2015;15:574.

2. Berkowitz SA, Seligman HK, Choudhry NK. Treat or eat: food insecurity, cost-related medication underuse, and unmet needs. Am J Med 2014;127:30310.e3.

3. Bonomi AE, Anderson ML, Rivara FP, Thompson RS. Health care utilization and costs associated with physical and nonphysical-only intimate partner violence. Health Serv Res 2009;44:1052-67.

4. Kushel MB, Gupta R, Gee L, Haas JS. Housing instability and food insecurity as barriers to health care among low-income Americans. J Gen Intern Med 2006;21:71-7.

5. Patel MR, Piette JD, Resnicow K, Kowalski-Dobson T, Heisler M. Social determinants of health, costrelated nonadherence, and cost-reducing behaviors among adults with diabetes: findings from the national health interview survey. Med Care 2016;54: 796-803.

6. Rivara FP, Anderson ML, Fishman P, et al. Healthcare utilization and costs for women with a history of intimate partner violence. Am J Prev Med 2007;32: 89-96.

7. Institute of Medicine. Capturing social and behavioral domains and measures in the electronic health record: phase 2. Washington, DC: National Academies Press; 2014.

8. Alley DE, Asomugha CN, Conway PH, Sanghavi DM. Accountable health communities-addressing social needs through Medicare and Medicaid. N Engl J Med 2016;374:8-11.

9. Secretary's Advisory Committee, Office of Disease Prevention and Health Promotion, US Department of Health \& Human Services. Healthy People 2020: an opportunity to address social determinants of health in the United States. July 26, 2010. Available from: http://www.healthypeople.gov/2010/hp2020/ advisory/SocietalDeterminantsHealth.htm. Accessed May 29, 2017.

10. Chung EK, Siegel BS, Garg A, et al. Screening for social determinants of health among children and families living in poverty: a guide for clinicians. Curr Probl Pediatr Adolesc Health Care 2016;46:135-53.

11. Fierman AH, Beck AF, Chung EK, et al. Redesigning health care practices to address childhood poverty. Acad Pediatr 2016;16:S136-46.

12. Frank DA, Casey PH, Black MM, et al. Cumulative hardship and wellness of low-income, young children: multisite surveillance study. Pediatrics 2010; 125:e1115-23.

13. Garg A, Butz AM, Dworkin PH, Lewis RA, Thompson RE, Serwint JR. Improving the management of family psychosocial problems at low-income children's well-child care visits: the WE CARE Project. Pediatrics 2007;120:547-58.

14. Giuse NB, Koonce TY, Kusnoor SV, et al. Institute of Medicine measures of social and behavioral determinants of health: a feasibility study. Am J Prev Med. 2017;52:199-206.

15. Hughes LS. Social determinants of health and primary care: intentionality is key to the data we collect and the interventions we pursue. J Am Board Fam Med 2016;29:297-300.

16. Kemper KJ. Self-administered questionnaire for structured psychosocial screening in pediatrics. Pediatrics 1992;89:433-6.

17. National Association of Community Health Centers. Research and data. Available from: http://www.nachc.org/research-and-data/. Accessed February 27, 2017.

18. Shin P, Alvarez C, Sharac J, et al. A profile of community health center patients: implications for policy. Issue brief. Menlo Park, CA: Henry J. Kaiser Family Foundation; 2013.

19. Health Resources and Services Administration. What is a health center? Available from: https:// bphc.hrsa.gov/about/what-is-a-health-center/index. html. Accessed February 27. 2017.

20. Michigan Primary Care Association. What are health centers? Lansing, MI: Michigan Primary Care Association; 2016. Available from: http://www. mpca.net/?page $=$ whatareHCs\#LAs. Accessed February 16, 2017.

21. Risk adjustment for socioeconomic status or other sociodemographic factors. Washington, DC: National Quality Forum; 2014. Available from: http:// www.qualityforum.org/Publications/2014/08/Risk_ Adjustment_for_Socioeconomic_Status_or_Other_ 
Sociodemographic_Factors.aspx. Accessed June 23, 2017.

22. Social determinants of health. Geneva: World Health Organization; 2017. Available from: http:// www.who.int/social_determinants/en/. Accessed February 27, 2017.

23. Elo S, Kyngas H. The qualitative content analysis process. J Adv Nurs 2008;62:107-15.

24. Krippendorff K. Content analysis: an introduction to its methodology. Thousand Oaks, CA: Sage; 2004.

25. Kroenke K, Spitzer RL, Williams JB. The PHQ-9: validity of a brief depression severity measure. J Gen Intern Med 2001;16:606-13.

26. Kroenke K, Spitzer RL, Williams JB. The Patient Health Questionnaire-2: validity of a two-item depression screener. Med Care 2003;41:1284-92.

27. Spitzer RL, Kroenke K, Williams JB, Löwe B. A brief measure for assessing generalized anxiety disorder: the GAD-7. Arch Intern Med 2006;166: 1092-7.

28. Council on Community Pediatrics; Committee on Nutrition. Promoting food security for all children. Pediatrics. 2015;136:e1431-8.

29. U.S. Preventive Services Task Force. Recommendations for primary care practice: published recommendations. Available from: https://www. uspreventiveservicestaskforce.org/BrowseRec/ Index. Accessed February 27, 2017.

30. ONC Health IT Certification Program: standards and certification regulations. Available from: https:// www.healthit.gov/policy-researchers-implementers/ standards-and-certification-regulations. Accessed February 27, 2017.

31. Mayfield D, McLeod G, Hall P. The CAGE questionnaire: validation of a new alcoholism screening instrument. Am J Psychiatry 1974;131:1121-3.
32. Patnode CD, Henderson JT, Thompson JH, Senger CA, Fortmann SP, Whitlock EP. Behavioral counseling and pharmacotherapy interventions for tobacco cessation in adults, including pregnant women. Ann Intern Med 2015;163:608-21.

33. Garg A, Toy S, Tripodis Y, Silverstein M, Freeman E. Addressing social determinants of health at well child care visits: a cluster RCT. Pediatrics 2015;135: e296-304.

34. Gottlieb LM, Hessler D, Long D, et al. Effects of social needs screening and in-person service navigation on child health: a randomized clinical trial. JAMA Pediatr 2016;170:e162521.

35. Garg A, Boynton-Jarrett R, Dworkin PH. Avoiding the unintended consequences of screening for social determinants of health. JAMA 2016;316:813-4.

36. Michigan Primary Care Association. Linking clinical care with community supports (linkages). Available from: http://www.mpca.net/?page=linkages. Accessed February 27, 2017.

37. State innovation model (SIM). Okemos: Michigan Public Health Institute; 2015. Available from: https://www.mphi.org/projects/state-innovationmodel-sim/. Accessed February 27, 2017.

38. Michigan pathways to better health. Okemos: Michigan Public Health Institute; 2015. Available from: https://www.mphi.org/projects/michigan-pathwaysto-better-health/. Accessed February 27, 2017.

39. Nath JB, Costigan S, Hsia RY. Changes in demographics of patients seen at federally qualified health centers, 2005-2014. JAMA Intern Med 2016;176: $712-4$.

40. Buntin MB, Ayanian JZ. Social risk factors and equity in medicare payment. N Engl J Med 2017;376: 507-10. 